\title{
Noradrenergic Excitation of Magnocellular Neurons in the Rat Hypothalamic Paraventricular Nucleus via Intranuclear Glutamatergic Circuits
}

\author{
Shabrine S. Daftary, ${ }^{1}$ Cherif Boudaba, ${ }^{2}$ Kriszta Szabó, ${ }^{2}$ and Jeffrey G. Tasker ${ }^{2,3}$ \\ ${ }^{1}$ Molecular and Cellular Biology Program, ${ }^{3}$ Neuroscience Training Program, and ${ }^{2}$ Department of Cell and Molecular \\ Biology, Tulane University, New Orleans, Louisiana 70118
}

Noradrenergic projections to the hypothalamus play a critical role in the afferent control of oxytocin and vasopressin release. Recent evidence for intrahypothalamic glutamatergic circuits prompted us to test the hypothesis that the excitatory effect of noradrenergic inputs on oxytocin and vasopressin release is mediated in part by local glutamatergic interneurons. The voltage response to norepinephrine $(30-300 \mu \mathrm{M})$ was tested with whole-cell recordings in putative magnocellular neurons of the paraventricular nucleus (PVN) in hypothalamic slices $(400 \mu \mathrm{m})$. Norepinephrine elicited an $\alpha_{1}$ receptor-mediated direct depolarization in $23 \%$ of the magnocellular neurons tested; however, the most prominent response, seen in $42 \%$ of the magnocellular neurons, was an $\alpha_{1}$ receptor-mediated increase in the frequency of EPSPs. The norepinephrine-induced increase in EPSPs was blocked by tetrodotoxin and by ionotropic glutamate receptor antagonists, suggesting that norepinephrine excited presynaptic glutamate neurons to cause an increase in

Magnocellular neurons of the hypothalamic paraventricular nucleus (PVN) and supraoptic nucleus (SON) are stimulated under certain physiological conditions to generate bursts of action potentials. Although intrinsic membrane ionic conductances capable of sustaining bursting activity have been characterized in magnocellular neurons (Bourque and Renaud, 1991; Legendre and Poulain, 1992), the synaptic mechanisms responsible for triggering bursts and for coordinating the bursting activity in response to specific sensory stimuli are less well understood.

Several lines of evidence suggest that noradrenergic inputs play a critical stimulatory role in the release of oxytocin and vasopressin under conditions of increased hormone demand. Oxytocinergic and vasopressinergic magnocellular neurons of the PVN and SON are contacted directly by noradrenergic synapses (Ginsberg et al., 1994; Michaloudi et al., 1997), and stimulation of the brainstem A1/A2 noradrenergic cell groups activates putative magnocellular neurons and causes oxytocin and vasopressin release (Day et al., 1984; Tanaka et al., 1985; Day and Sibbald, 1988;

\footnotetext{
Received July 16, 1998; revised Sept. 8, 1998; accepted Sept. 22, 1998.

This work was funded by National Institute of Neurological Disorders and Stroke Grant NS31187. S.S.D. was partially supported by a predoctoral fellowship from the Louisiana American Heart Association. We thank Dr. H. Gainer for supplying oxytocin and vasopressin antibodies, Dr. A. Fancsik for his help with the computer analyses, and Drs. Shi Di and Andrei Belousov for their critical reading of this manuscript.

S.S.D. and C.B. contributed equally to this study

Correspondence should be addressed to Jeffrey Tasker, Department of Cell and Molecular Biology, Tulane University, New Orleans, LA 70118.

Copyright (C) 1998 Society for Neuroscience $\quad 0270-6474 / 98 / 1810619-10 \$ 05.00 / 0$
}

spike-mediated transmitter release. The increase in EPSPs also was observed in a surgically isolated PVN preparation $(64 \%$ of cells) and with microdrop applications of norepinephrine $(1 \mathrm{mM}$, $33 \%$ of cells) and glutamate $(0.5-1 \mathrm{~mm}, 28 \%)$ in the PVN, indicating that the norepinephrine-sensitive presynaptic glutamate neurons are located within the PVN. Biocytin injection and subsequent immunohistochemical labeling revealed that both oxytocin and vasopressin neurons responded to norepinephrine. Our data indicate that magnocellular neurons of the PVN receive excitatory inputs from intranuclear glutamatergic neurons that express $\alpha_{1}$-adrenoreceptors. These glutamatergic interneurons may serve as an excitatory relay in the afferent noradrenergic control of oxytocin and vasopressin release under certain physiological conditions.

Key words: hypothalamus; paraventricular nucleus; oxytocin; vasopressin; norepinephrine; adrenoreceptors; glutamate

Kim et al., 1989). This effect is blocked by 6-hydroxydopamine lesion of the hypothalamic noradrenergic projections (Day et al., 1984). Intracerebroventricular or local hypothalamic injection of norepinephrine or $\alpha_{1}$-receptor agonists results in the release of oxytocin (Bridges et al., 1976; Tribollet et al., 1978) and vasopressin (Benetos et al., 1986; Willoughby et al., 1987). Similarly, $\alpha_{1}$-receptor agonists applied to hypothalamic explants causes depolarization and spike generation in SON magnocellular neurons and leads to an increase in oxytocin and vasopressin release (Armstrong et al., 1986; Randle et al., 1986a,b).

These noradrenergic afferents may be part of the ascending sensory pathways responsible for selectively activating the oxytocin neurons during parturition and reflex milk ejection and the vasopressin neurons during hemorrhage. During parturition, the norepinephrine concentration in the SON rises prior to and in parallel with increases in blood levels of oxytocin (Herbison et al., 1997). Blockade of noradrenergic inputs to the hypothalamus with 6-hydroxydopamine lesion or with $\alpha_{1}$-adrenoreceptor antagonists inhibits the reflex release of oxytocin associated with milk ejection (Tribollet et al., 1978; Clarke et al., 1979; Crowley et al., 1987). Similarly, reflex vasopressin release in response to unloading of arterial baroreceptors is accompanied by a rise in the concentration of norepinephrine in the PVN (Van Huysse and Bealer, 1991), which is caused by the activation of A1 noradrenergic projections (Day and Renaud, 1984).

Several studies suggest a possible role of glutamate in the triggering mechanism for oxytocin release. Glutamate levels in the SON have been found to rise abruptly just before parturition 
and to decline before delivery is terminated (Herbison et al., 1997), and intracerebroventricular injection of a glutamate receptor antagonist blocks the suckling-induced release of oxytocin (Parker and Crowley, 1993a). We recently found evidence for excitatory synaptic inputs to magnocellular neurons of the PVN and SON from intrahypothalamic glutamate neurons (Boudaba et al., 1997). The current study was conducted to determine whether the excitatory effect on oxytocin and vasopressin release of noradrenergic inputs is mediated by local glutamatergic circuits.

A preliminary account of this data has been published previously in abstract form (Daftary et al., 1996).

\section{MATERIALS AND METHODS}

Slice preparation. Male Sprague Dawley rats (50-150 gm; Charles River, Wilmington, MA) were deeply anesthetized with pentobarbitol sodium $(50 \mathrm{mg} / \mathrm{kg}$ body weight) and decapitated. The brain was quickly and gently removed from the cranial cavity and immersed in cooled $\left(1-2^{\circ} \mathrm{C}\right)$, oxygenated $\left(100 \% \mathrm{O}_{2}\right)$ artificial CSF (aCSF). The composition of the aCSF was (in mM): $140 \mathrm{NaCl}, 3 \mathrm{KCl}, 1.3 \mathrm{MgSO}_{4}, 1.4 \mathrm{NaH}_{2} \mathrm{PO}_{4}, 2.4$ $\mathrm{CaCl}_{2}, 11$ glucose, and 5 HEPES; $\mathrm{pH}$ was adjusted to $7.2-7.4$ with $\mathrm{NaOH}$. The hypothalamus was blocked with a razor, and $400 \mu \mathrm{m}$ hypothalamic slices were sectioned in the coronal plane using a vibrating microtome (World Precision Instruments, Sarasota, FL). Two slices containing the PVN were identified, and a single slice was transferred immediately to a ramp-style, interface recording chamber where it was perfused with humidified, oxygenated aCSF maintained at $32-34^{\circ} \mathrm{C}$ and allowed to equilibrate for at least $1 \mathrm{hr}$ before recordings were started. The second slice was stored submerged in a holding chamber in oxygenated aCSF at room temperature until it was used. In some experiments, the PVN was surgically isolated from the rest of the slice under a dissecting microscope using a scalpel.

Electrophysiological methods. Sharp microelectrodes were made from microfilament glass capillaries [1.0 mm outer diameter (o.d.), $0.6 \mathrm{~mm}$ inner diameter (i.d); World Precision Instruments], and patch pipettes were pulled from borosilicate glass $(1.65 \mathrm{~mm}$ o.d., $1.2 \mathrm{~mm}$ i.d.; KG-33; Garner Glass, Claremont, CA) using a Flaming-Brown P-97 micropipette puller (Sutter Instruments, Novato, CA). Sharp electrodes were filled with $2 \mathrm{M}$ potassium acetate. Patch pipettes were filled with a solution containing (in $\mathrm{mM}$ ): 120 potassium gluconate, $10 \mathrm{HEPES}, 1$ $\mathrm{NaCl}, 1 \mathrm{CaCl}_{2}, 1 \mathrm{MgCl}_{2}, 2 \mathrm{Mg}$-ATP, $0.3 \mathrm{Na}-\mathrm{GTP}$, and 10 EGTA; pH was adjusted to 7.2-7.4 with $\mathrm{KOH}$. The osmolarity of the patch solution was made hyperosmotic (290-310 M $\Omega / 1)$ with $20 \mathrm{mM}$ D-sorbitol to reduce series resistance.

The slice was transilluminated in the recording chamber, and the recording electrode was positioned in the magnocellular PVN under visual guidance using a dissecting microscope. The electrode was lowered through the slice by 2 - to $4-\mu \mathrm{m}$-steps with a piezoelectric microdrive (Nanostepper, Adams \& List, Westbury, NY). Recordings were performed in current-clamp mode using an Axoclamp 2A amplifier (Axon Instruments, Foster City, CA) and were monitored continuously on a digital storage oscilloscope (Hitachi, Tokyo, Japan). Data were converted to digital video format (Neurocorder, Neurodata Instruments, New York, NY) and stored on videotape for off-line analysis. Episodes of spontaneous EPSPs $(30-60 \mathrm{sec}) 1 \mathrm{~min}$ before and 8-9 min into norepinephrine application were amplified $10 \times$, filtered at $1 \mathrm{kHz}$ with a filter/amplifier (Cygnus Technology, Delaware Water Gap, PA), and digitized at 2-4 $\mathrm{kHz}$ with a TL-1 interface and the pClamp 6.1 suite of software (Axon Instruments). EPSP responses to $100 \mu \mathrm{M}$ norepinephrine were analyzed for changes in frequency and amplitude with the Datapac program (Run Technologies, Laguna Hills, CA). Cumulative probability distributions of EPSP amplitude and instantaneous frequency were generated using an in-house program and compared with the Kolmogorov-Smirnov test. Population means were compared with the Student's paired $t$ test and the Wilcoxon signed rank test for frequency and amplitude values, respectively. Probability values $<0.05$ were considered significant. Means are expressed as $\pm \mathrm{SE}$.

Drug application. Norepinephrine, adrenoreceptor antagonists, tetrodotoxin (TTX), glutamate and glutamate receptor antagonists were dissolved in aCSF and either bath-applied, or, where indicated, norepinephrine and glutamate microdrops were applied by pressure on the surface of the slice using a picospritzer (General Valve, Fairfield, NJ). Norepinephrine (Sigma, St. Louis, MO) was bath-applied for 5-15 min at concentrations ranging from $30 \mu \mathrm{M}$ to $300 \mu \mathrm{M}$. Norepinephrine $(1 \mathrm{mM})$ and glutamate $(0.5-1 \mathrm{~mm})$ microdrops were applied under visual control at one or more sites in the PVN using a patch pipette with a broken tip positioned with a micromanipulator (Newport Corporation, Irvine, CA). Janus green $(0.1 \%)$ was added to the norepinephrine and glutamate microdrop solutions to monitor visually the spread of the drops. Adrenoreceptor antagonists included the $\alpha_{1}$-adrenoreceptor antagonist prazosin hydrochloride $(10 \mu \mathrm{M})$ and the $\beta$-adrenoreceptor antagonist propranolol hydrochloride $(10 \mu \mathrm{M})$ (Research Biochemicals International, Natick, MA); glutamate receptor antagonists included the NMDA receptor antagonist D,L-2-amino-5-phosphonovalerate (AP5) $(100 \mu \mathrm{M})$ and the non-NMDA receptor antagonist 5,6-dinitroquinoxaline-2, 3-dione (DNQX; $50 \mu \mathrm{M}$ ) (Tocris Cookson, Ballwin, MO). Adrenoreceptor and glutamate receptor antagonists were bath-applied for $15 \mathrm{~min}$ before the reapplication of norepinephrine. Tetrodotoxin (1.5-3 $\mu \mathrm{M})$ (Sigma) was used to block voltage-gated sodium channels and spike-mediated transmitter release. Stock solutions $(10 \mathrm{mM})$ of prazosin hydrochloride and propranolol hydrochloride were prepared in aCSF and stored in the dark at $-20^{\circ} \mathrm{C}$ until use.

Biocytin histology and peptide immunohistochemistry. Biocytin was added to microelectrodes $(1 \%)$ and to patch pipettes $(0.3-0.5 \%)$ as an intracellular marker. The biocytin leaked into the recorded cells during patch recordings, or in the case of microelectrode recordings was iontophoresed intracellularly at the end of experiments by passing negative current pulses $(-250 \mathrm{pA}, 250 \mathrm{msec}, 2 \mathrm{~Hz})$ for 5-10 min.

After experiments, slices were removed from the recording chamber and fixed overnight in $4 \%$ paraformaldehyde in $0.1 \mathrm{M} \mathrm{PBS}$ at $4^{\circ} \mathrm{C}$. They were then sectioned on a freezing microtome at 20-25 $\mu \mathrm{m}$, and the biocytin-injected cells were labeled by incubating the sections for $4 \mathrm{hr}$ in streptavidin-conjugated 7-amino-4-methyl-coumarin-3-acetic acid (AMCA; Molecular Probes, Eugene, OR). The AMCA was diluted 1:300 in $0.1 \mathrm{M}$ PBS containing $0.5 \%$ Triton X-100. Sections were scanned under a fluorescence microscope using a UV/420K filter combination to detect the presence of biocytin-filled, AMCA-labeled neurons.

Sections containing the AMCA-labeled cells were placed in $2 \%$ normal sheep serum in $0.1 \mathrm{M}$ PBS for $15 \mathrm{~min}$. To determine whether the stained cells were oxytocin or vasopressin magnocellular neurons, we used a mixture of a rabbit polyclonal antibody to oxytocin (VA-10) and a mouse monoclonal antibody to vasopressin-associated neurophysin (PS41) on the same section. Both antibodies were kindly provided by Dr. H. Gainer (National Institutes of Health, Bethesda, MD) (Ben-Barak et al., 1985; Altstein et al., 1988). The polyclonal oxytocin antibody (1:2000) and the monoclonal vasopressin-associated neurophysin antibody $(1: 200)$ were applied together for $36 \mathrm{hr}$ at $4^{\circ} \mathrm{C}$ in $0.1 \mathrm{~m}$ PBS $+1 \%$ normal sheep serum and $0.2 \%$ sodium azide. After treatment with the primary antibodies, sections were rinsed with $0.1 \mathrm{M}$ PBS, incubated for $1 \mathrm{hr}$ in a mixture of anti-rabbit IgG conjugated to fluorescein isothiocyanate (FITC, 1:100; Vector Labs, Burlingame, CA) and anti-mouse IgG conjugated to rhodamine (1:100; Jackson ImmunoResearch Labs, West Grove, PA), and rinsed again in $0.1 \mathrm{M}$ PBS. They then were mounted, coverslipped, and examined under $450-490 \mathrm{~nm}$ excitation $/ 515 \mathrm{~nm}$ barrier filters to detect the FITC-labeled oxytocin neurons and 515-560 nm excitation/580 $\mathrm{nm}$ barrier filters to see the rhodamine-labeled vasopressin neurons. Recorded cells were positively identified as either oxytocinergic or vasopressinergic only if they labeled positive for one of the two antibodies and negative for the other. No cells were found to be positively labeled for both antibodies, confirming the specificity of the antibodies. We have tested the polyclonal oxytocin antibody (VA-10) for specificity using preabsorption controls (Boudaba et al., 1996).

\section{RESULTS}

Putative magnocellular neurons of the PVN were distinguished from putative parvocellular neurons during recordings based on specific electrophysiological properties (Hoffman et al., 1991; Tasker and Dudek, 1991). In particular, magnocellular neurons have a prominent transient outward rectification, a relatively short membrane time constant, and linear current-voltage relations.

A total of 136 putative magnocellular neurons were recorded in this study: 122 cells were tested for their response to norepinephrine and 14 cells for their response to glutamate microdrops. Thirteen of the cells were recorded with sharp electrodes to control for washout of the norepinephrine signal; these cells 
showed the same responses to norepinephrine as the rest of the cells that were recorded with patch electrodes. The neurons recorded with sharp electrodes had a mean membrane potential of $-54 \pm 2 \mathrm{mV}$ (SE), input resistance of $215 \pm 24 \mathrm{M} \Omega$, and action potential amplitude of $65 \pm 2 \mathrm{mV}$ (threshold-peak). The cells recorded with patch electrodes had a mean membrane potential of $-66 \pm 1 \mathrm{mV}$ (corrected for a $-11 \mathrm{mV}$ junction potential), input resistance of $871 \pm 65 \mathrm{M} \Omega$, and action potential amplitude of $66 \pm 1 \mathrm{mV}(n=58)$. The patch solution was weakly hyperosmotic, which resulted in a hyperpolarized resting potential. Application of norepinephrine caused a direct depolarization or an increase in EPSPs, or both, in the majority $(60 \%)$ of magnocellular neurons recorded.

\section{Direct effect of norepinephrine}

Norepinephrine was considered to have a presumptive direct, postsynaptic effect if it resulted in a sustained change in membrane potential of at least $3 \mathrm{mV}$ that was reversed with washout. Bath application of norepinephrine at concentrations of 30-300 $\mu \mathrm{M}$ elicited a reversible depolarization $(7.27 \pm 0.6 \mathrm{mV})$ in 18 of 90 (20\%) putative magnocellular neurons tested (Fig. 1A). The depolarization occurred within $\sim 3-7$ min of norepinephrine introduction into the recording chamber and was accompanied by a decrease in input resistance $(15 \pm 2 \%)$ in 4 of 16 cells tested. The response was not blocked by TTX $(1.5-3 \mu \mathrm{M}, n=3)$, suggesting that it was not mediated by an increase in spike-mediated transmitter release. It was blocked or reduced by the $\alpha_{1}$ adrenoreceptor antagonist prazosin $(10 \mu \mathrm{M}, n=4)$ but was not affected by the $\beta$-adrenoreceptor antagonist propranolol $(10 \mu \mathrm{M}$, $n=3$ ) (data not shown). The direct response to norepinephrine was qualitatively similar to that described in magnocellular neurons of the supraoptic nucleus (Randle et al., 1986a) and was not investigated further in this study.

\section{Norepinephrine activation of presynaptic glutamate neurons}

Bath application of norepinephrine (30-300 $\mu \mathrm{M})$ caused a large increase in EPSPs in 38 of $90(42 \%)$ putative magnocellular neurons tested in the whole slice (Fig. $1 B$ ). An increase in EPSPs was indicated by a qualitatively detectable rise in the frequency (i.e., of at least 10-20\%) of positive-going synaptic potentials recorded at resting membrane potential. The increase in EPSPs occurred $\sim 7-8 \mathrm{~min}$ after introduction of the norepinephrine into the recording chamber, was maintained throughout the application, and reversed within 10-33 min of washout of the norepinephrine. Neurons responding to $100 \mu \mathrm{M}$ norepinephrine were selected for EPSP frequency and amplitude analysis. Norepinephrine caused a significant increase in both the frequency $(p<$ $0.001 ; n=22$; Student's paired $t$ test) and the amplitude $(p<$ $0.05 ; n=10$; Wilcoxon signed rank test) of spontaneous EPSPs collected in 30-60 sec episodes. This was seen in individual cells as a significant shift in the cumulative EPSP frequency and amplitude distributions ( $p<0.01 ; n=5$; Kolmogorov-Smirnov test) (Fig. 1C). The mean percentage increases in EPSP frequency $(n=22)$ and amplitude $(n=10)$ were 145 and $52 \%$, respectively (Fig. 1D). The marked rise in the frequency of EPSPs suggests that norepinephrine increases the probability of transmitter release from presynaptic excitatory neurons. The moderate increase in EPSP amplitude suggests that it may also modulate the postsynaptic responsiveness of the magnocellular neurons, although this effect is less robust. No apparent desensitization of the synaptic response was observed during the norepi- nephrine application or with a second application of norepinephrine $(n=3)$. Of the 38 cells that showed an increase in EPSPs, eight cells also depolarized in response to norepinephrine.

The norepinephrine-induced increase in EPSPs was blocked by the $\alpha_{1}$-adrenoreceptor antagonist prazosin hydrochloride $(10 \mu \mathrm{M})$ in six of six cells tested (Fig. 2). The $\beta$-receptor antagonist propranolol hydrochloride $(10 \mu \mathrm{M})$ failed to block the EPSPs elicited by norepinephrine $(n=5)$. Thus, norepinephrine appears to activate presynaptic excitatory neurons by acting at $\alpha_{1}$-receptors.

To test whether norepinephrine caused an increase in EPSPs by acting at presynaptic neurons, we applied TTX $(1.5-3 \mu \mathrm{M})$ to block spike-mediated transmitter release. Bath application of TTX blocked completely the norepinephrine-evoked increase in EPSPs in eight of eight cells tested (Fig. 3). This indicated that the increased EPSPs were caused by an increase in spike-evoked transmitter release and suggested that norepinephrine was acting at receptors at the somatic/dendritic region of presynaptic excitatory neurons. In the cells that responded with an increase in EPSPs, norepinephrine had no apparent effect on the depolarizing voltage responses to positive current pulses $(n=4)$, suggesting that norepinephrine was not acting postsynaptically to amplify spontaneous EPSPs by enhancing or attenuating voltagegated currents.

That norepinephrine application led to an increase in the frequency of fast EPSPs suggested that it was acting to stimulate presynaptic glutamate neurons, causing an increase in glutamate release and activation of postsynaptic ionotropic glutamate receptors. We tested this hypothesis by bath applying the NMDA and non-NMDA receptor antagonists AP5 and DNQX, respectively, to block ionotropic glutamate receptors. In cells that had shown a norepinephrine-evoked increase in EPSPs, AP5 and DNQX blocked completely the synaptic response to norepinephrine in six of six cells tested (Fig. 4). Thus, the presynaptic neurons that responded to norepinephrine with a spike-mediated increase in transmitter release onto PVN magnocellular neurons were glutamatergic, and the synaptic responses were caused by glutamate activation of ionotropic glutamate receptors.

\section{Intranuclear localization of the presynaptic glutamate neurons}

The TTX experiments suggested that the presynaptic glutamate neurons were present and intact in our slices, because the norepinephrine was probably acting at the somatic/dendritic regions of the presynaptic cells to cause action potential generation. This, along with the relatively high percentage of magnocellular neurons $(42 \%)$ in our slices that responded to norepinephrine with an increase in EPSPs, indicated that the presynaptic glutamate neurons were in close proximity to the magnocellular neurons, possibly inside the PVN.

We conducted three experiments to test this hypothesis. The first experiment tested the effects of norepinephrine on the incidence of EPSPs in PVN magnocellular neurons in a slice preparation in which the PVN was surgically isolated by cutting away and removing the rest of the slice. In this preparation, bath application of norepinephrine caused an increase in the frequency of EPSPs in 7 of $11(64 \%)$ putative magnocellular neurons tested (Fig. 5). Next, glutamate (0.5-1 mm) was applied as microdrops directly into the PVN to stimulate neurons focally within the PVN without activating axons of passage (Christian and Dudek, 1988). Glutamate microstimulation led to an increase in 

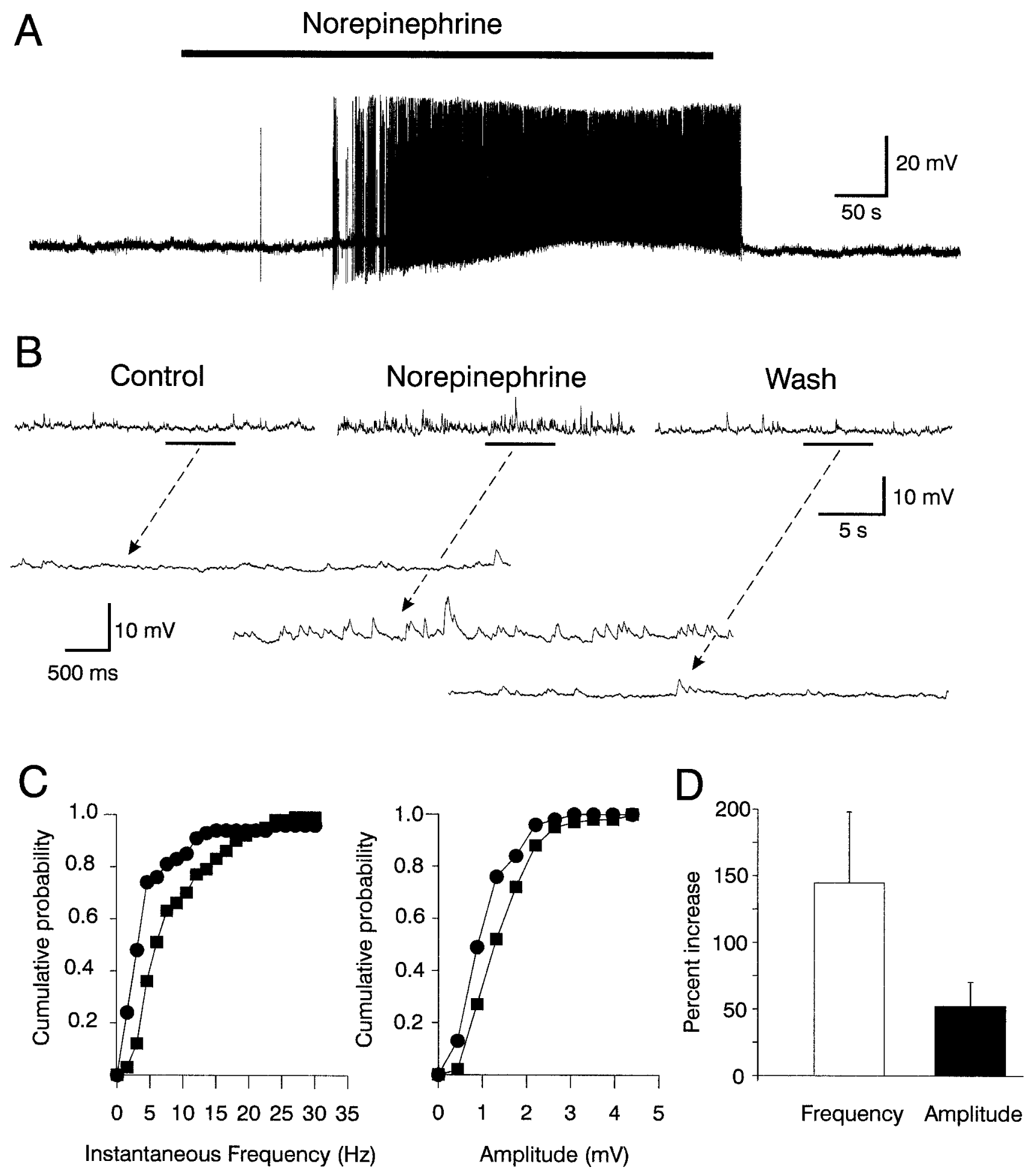

Figure 1. Norepinephrine responses of PVN magnocellular neurons. A, Direct depolarization elicited by norepinephrine. A putative magnocellular neuron responded to bath application of norepinephrine $(300 \mu \mathrm{M})$ with a $10 \mathrm{mV}$ depolarization; no apparent change in input resistance was seen (data not shown). This cell had a resting membrane potential of $-54 \mathrm{mV}$. Positive spikes are action potentials, which were truncated by digital filtering. Bar indicates the duration of the norepinephrine application. $B$, Increased EPSPs in norepinephrine. Bath application of norepinephrine (100 $\mu \mathrm{M})$ elicited an increase in EPSPs (Norepinephrine) with a latency of $\sim 8 \mathrm{~min}$ in a putative magnocellular neuron recorded at resting potential. The response reversed after $20 \mathrm{~min}$ in normal aCSF (Wash). This cell had a resting membrane potential of $-60 \mathrm{mV}$. Bottom traces are expanded recordings of the periods in the top traces indicated by the bars. $C$, Cumulative probability plots of the EPSP frequency and amplitude distributions in a representative neuron in control aCSF $(\bullet)$ and during norepinephrine application $(\boldsymbol{\square})$. There is a significant shift toward higher instantaneous frequencies and larger amplitudes of the EPSPs in norepinephrine ( $p<0.01 ; n=5$; Kolmogorov-Smirnov test). $D$, Changes in mean frequency and amplitude of EPSPs in norepinephrine. Mean frequencies and amplitudes were calculated in control medium and in $100 \mu \mathrm{M}$ norepinephrine. These values were averaged, and the differences were expressed as percentage increase in norepinephrine. There was a 147\% average increase in the mean frequency $(n=22)$ and a $53 \%$ average increase in the mean amplitude $(n=10)$ of EPSPs in norepinephrine. 


\section{Control}

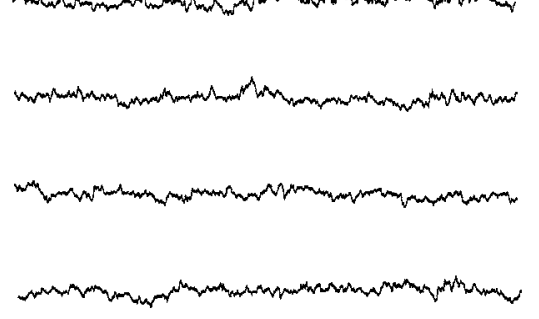

Norepinephrine

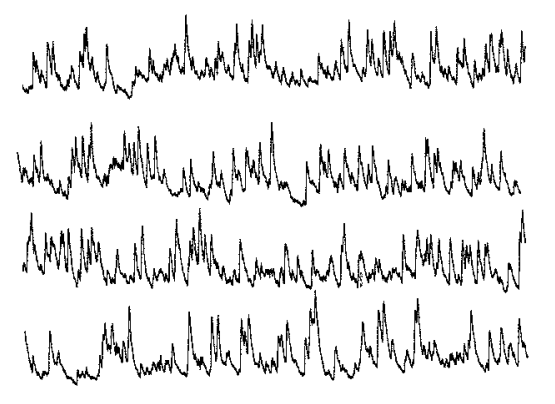

\section{Norepinephrine in Prazosin}

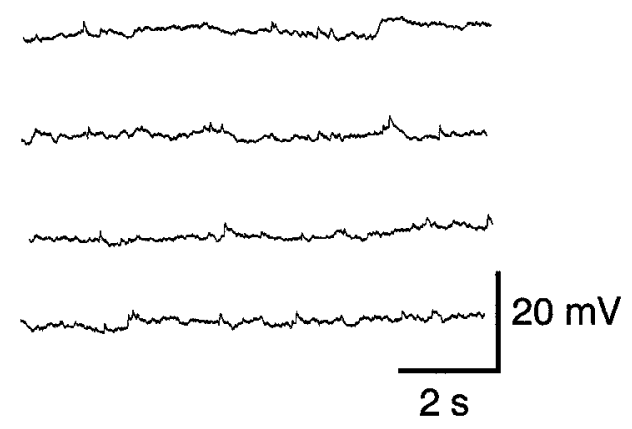

Figure 2. The norepinephrine-induced increase in EPSPs is mediated by $\alpha_{1}$-receptor activation. Bath application of norepinephrine (100 $\left.\mu \mathrm{M}\right)$ caused a reversible increase in the frequency of EPSPs (Norepinephrine) in a putative magnocellular neuron. A second norepinephrine application in the presence of the $\alpha_{1}$-receptor antagonist, prazosin hydrochloride (10 $\mu \mathrm{M}$; Norepinephrine in Prazosin), failed to elicit the increase in EPSPs, suggesting that the response was mediated by the activation of $\alpha_{1}$-adrenoreceptors. This cell had a resting membrane potential of $-64 \mathrm{mV}$. The cell was subsequently found to be immunopositive for oxytocin and immunonegative for vasopressin, which is shown in Figure 7.

\section{Control}

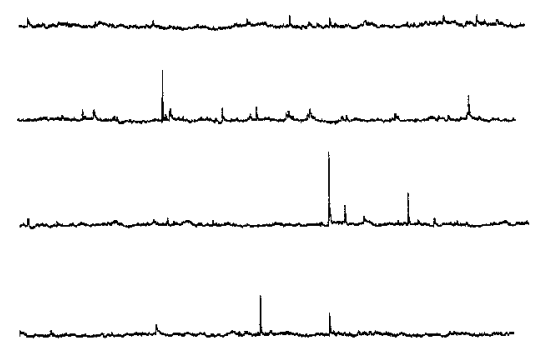

Norepinephrine

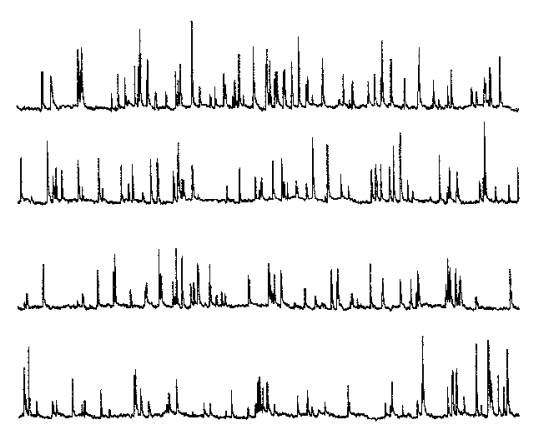

\section{Norepinephrine in TTX}
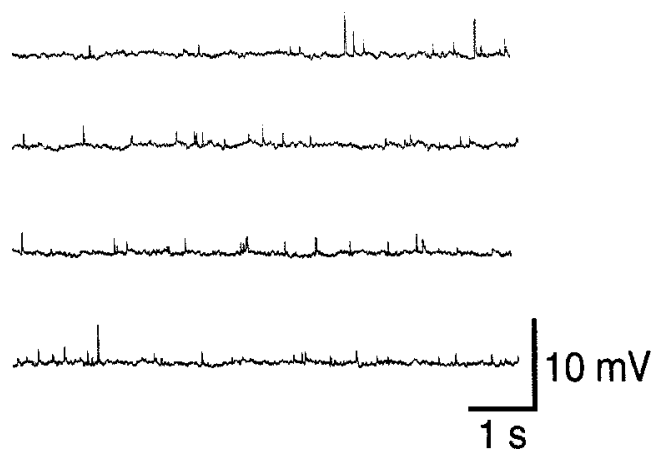

Figure 3. The norepinephrine-induced increase in EPSPs is caused by activation of local presynaptic excitatory neurons. Norepinephrine (100 $\mu \mathrm{M})$ elicited a reversible increase in EPSPs (Norepinephrine) in a putative magnocellular neuron recorded at a resting membrane potential of $-55 \mathrm{mV}$. The norepinephrine-induced EPSPs were blocked by TTX (Norepinephrine in TTX), suggesting that they were caused by the activation of presynaptic neuronal somata/dendrites, resulting in an increase in spike-mediated release of excitatory neurotransmitter. That the presynaptic neurons were intact in the slice suggests that they were cells with short axonal projections located in relative proximity to the recorded cell.

EPSPs in 4 of $14(28 \%)$ putative magnocellular neurons tested (data not shown), suggesting the presence of intranuclear excitatory circuits. Finally, to determine whether these intranuclear excitatory circuits were the same circuits as those activated by bath application of norepinephrine, we applied microdrops of norepinephrine $(1 \mathrm{~mm})$ directly into the PVN. Norepinephrine microdrops also elicited an increase in EPSPs in 7 of $21(33 \%)$ putative magnocellular neurons tested (Fig. 6). Four of the seven $(57 \%)$ neurons also exhibited a depolarization in response to norepinephrine. The results of these experiments all point to the presence of glutamate neurons within or very closely apposed to the PVN that are excited by norepinephrine and that send intranuclear projections to magnocellular neurons. Stimulation of these local glutamate interneurons via $\alpha_{1}$-receptor activation results in an increase in excitatory synaptic input to magnocellular neurons.

\section{Immunohistochemical identification of magnocellular neurons}

A total of 20 putative magnocellular neurons were recovered after biocytin labeling and oxytocin/vasopressin immunohistochemical processing with antibodies to oxytocin and vasopressin neurophysin. Only those cells that were immunopositive for one and negative for the other of the two antibodies were counted. Of the eight cells that responded to norepinephrine with a depolarization, five were immunopositive for oxytocin and immunonegative for vasopressin, and three were immunopositive for vasopressin and immunonegative for oxytocin. Of the 12 cells that responded to norepinephrine with an increase in EPSPs, three were immunopositive for vasopressin and immunonegative for oxytocin (Fig. 7A), and nine were immunopositive for oxytocin and immunonegative for vasopressin (Fig. $7 B$ ). These results suggest that both oxytocin and vasopressin magnocellular neu- 


\section{Control}
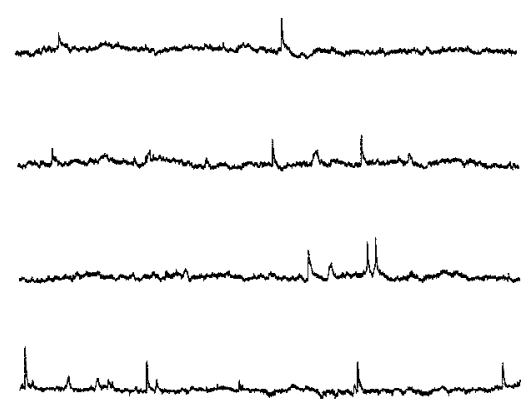

Norepinephrine

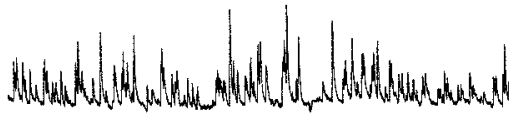

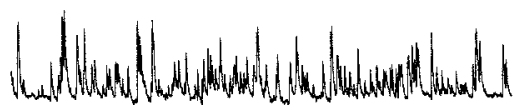

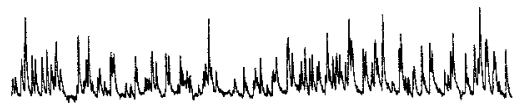

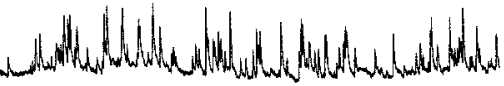

Norepinephrine

in DNQX, AP5
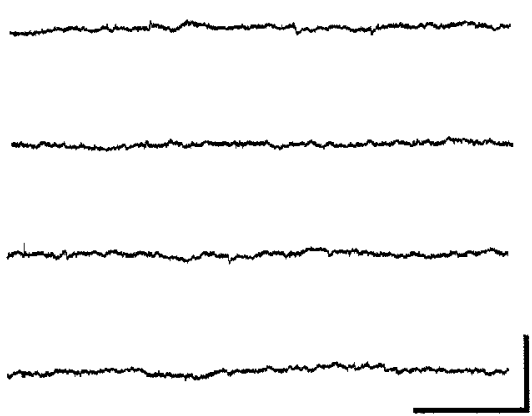

$10 \mathrm{mV}$

$1 \mathrm{~s}$

Figure 4. The norepinephrine-induced increase in EPSPs is mediated by presynaptic glutamate neurons. Norepinephrine (100 $\mu \mathrm{M})$ caused an increase in the frequency of EPSPs in a magnocellular neuron. The response reversed after $21 \mathrm{~min}$ in wash (data not shown). The EPSPs elicited by norepinephrine were completely blocked by the NMDA and non-NMDA receptor antagonists AP5 (100 $\mu \mathrm{M})$ and DNQX (50 $\mu \mathrm{M})$, suggesting that they were mediated by the release of glutamate and the activation of ionotropic glutamate receptors. This cell had a resting membrane potential of $-63 \mathrm{mV}$.

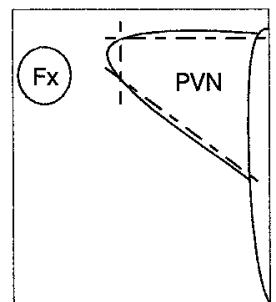

\section{Control}

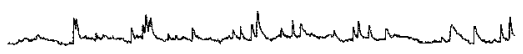

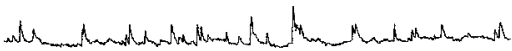

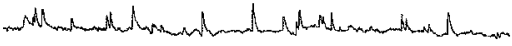

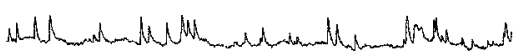

Norepinephrine

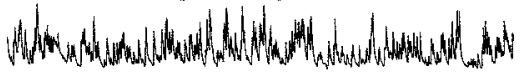

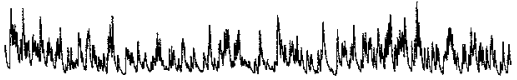

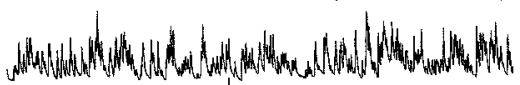

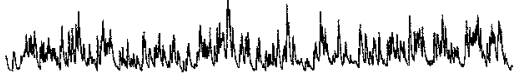

Wash

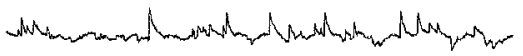

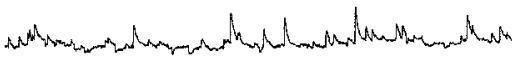

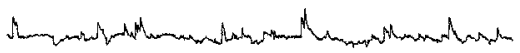

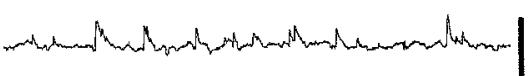

$10 \mathrm{mV}$

Figure 5. Norepinephrine-evoked increase in EPSPs in an isolated PVN preparation. A putative magnocellular neuron was recorded in a slice preparation in which the PVN had been surgically isolated from the rest of the slice (inset). Bath application of norepinephrine (100 $\mu \mathrm{M})$ elicited a robust increase in the frequency of EPSPs (Norepinephrine), and the response reversed after 20 min in regular aCSF (Wash). This cell had a resting membrane potential of $-65 \mathrm{mV}$. PVN, Paraventricular nucleus; $F x$, fornix.

rons express functional $\alpha_{1}$-adrenoreceptors and receive intranuclear excitatory synaptic inputs from glutamate neurons located within the PVN.

\section{DISCUSSION}

Despite the apparent prominent role of norepinephrine in the control of oxytocin and vasopressin neuronal activity and hormone release, little is known about the mechanisms or the locus of the excitatory actions of norepinephrine in the PVN. This is the first study to characterize the physiological actions of norepinephrine in identified oxytocin and vasopressin neurons. Electrophysiological distinction between magnocellular and parvocellular neurons combined with intracellular dye injection and post hoc immunohistochemical double-labeling with selective antibodies for oxytocin and vasopressin-associated neurophysin provided a reliable means of identifying PVN magnocellular neurons.

Norepinephrine excited PVN magnocellular neurons either directly by membrane depolarization or indirectly via an increase in EPSPs. The depolarizing effect of norepinephrine was mediated by $\alpha_{1}$-receptor activation and was similar qualitatively to that described in magnocellular neurons of the SON (Randle et al., 1986a), and it was therefore not characterized in further detail in this study. The norepinephrine-induced increase in synaptic activity in magnocellular neurons, on the other hand, has not been reported previously and was the primary focus of this study.

Several observations indicate that the increase in EPSPs was caused mainly by a presynaptic action of norepinephrine. The norepinephrine-induced synaptic response was TTX-sensitive 


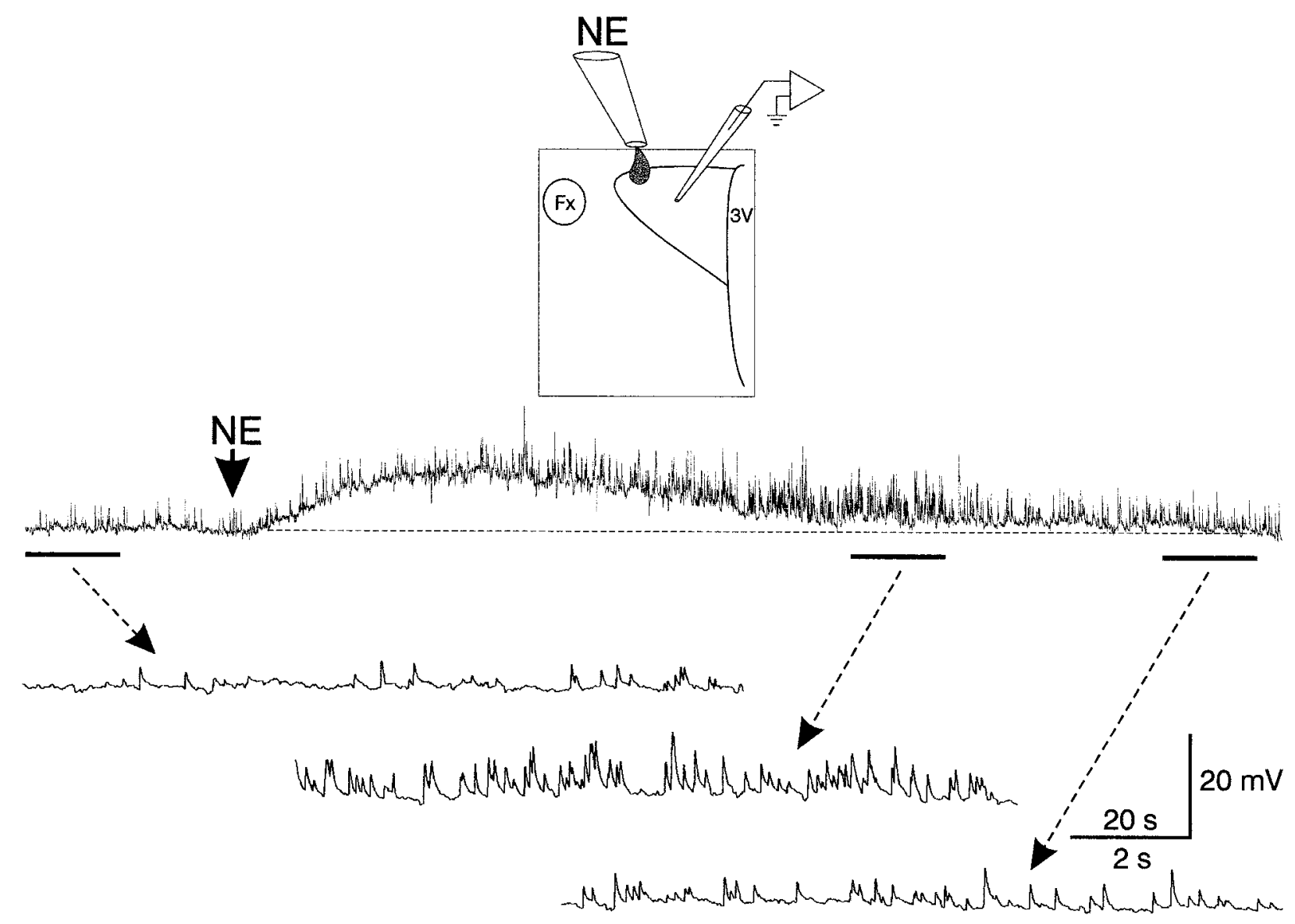

Figure 6. Norepinephrine microdrops in the PVN evoked an increase in EPSPs. Norepinephrine microdrop application in the PVN (inset) elicited a robust increase in EPSPs recorded in a putative magnocellular neuron. This effect was accompanied by a depolarization of the cell from its resting membrane potential of -64 to $-55 \mathrm{mV}$. Bottom traces are expanded recordings of the periods in the top traces indicated by the bars. Top time calibration applies to top trace; bottom time calibration applies to expanded traces. NE, Norepinephrine; $P V N$, paraventricular nucleus; $F x$, fornix; $3 V$, third ventricle.

and was characterized by a robust increase in the frequency of EPSPs, suggestive of an enhanced probability of transmitter release. The majority of the cells that responded to norepinephrine with an increase in EPSPs (79\%) did not show any change in resting membrane potential or input resistance, indicating that norepinephrine was not acting on postsynaptic conductances active at resting potential. Similarly, norepinephrine had no effect on depolarizing voltage responses to positive current pulses in these cells, suggesting that it was not acting on postsynaptic voltage-gated conductances. However, the norepinephrineinduced increase in EPSP amplitudes suggests that norepinephrine may also have a postsynaptic modulatory effect on ionotropic glutamate receptor-mediated currents in these cells, but these actions of norepinephrine appear less robust than the presynaptic actions.

The norepinephrine-induced increase in EPSPs was most likely due to the activation of receptors located in the somatic/dendritic region of presynaptic neurons. The response was blocked completely by TTX and was therefore dependent on spike generation, presumably at the initial segment of the axon. That the response was not mediated by modulation of spike-dependent conductances at presynaptic terminals or preterminal axons (Lena et al., 1993 ) is suggested by our experiments involving glutamate microdrops, whose excitatory actions should be restricted to the somatic/dendritic membrane (Christian and Dudek, 1988;
Schrader and Tasker, 1997). Glutamate microdrops elicited EPSPs in a proportion of PVN cells comparable to the proportion of cells that responded to norepinephrine microdrops (28 vs 33\%, respectively). If the glutamate and norepinephrine microdrops acted on the same presynaptic cells, which seems a likely possibility, then the norepinephrine, like the glutamate, was probably acting at presynaptic somatic/dendritic receptors.

Our data suggest that norepinephrine activated presynaptic glutamate interneurons located within, or in very close proximity to, the PVN. The fast kinetics of the EPSPs and their sensitivity to ionotropic glutamate receptor antagonists indicate that they were mediated by glutamate release. The glutamatergic synaptic inputs originated in neurons located inside the PVN or very close to the periphery of the nucleus because both norepinephrine microdrops applied within the PVN and bath application of norepinephrine in a surgically isolated PVN elicited EPSPs. The percentage of cells responding to norepinephrine with augmented EPSPs increased in the isolated PVN preparation, from $\sim 40$ to $>60 \%$, which could be explained by excitatory actions of norepinephrine on perinuclear inhibitory neurons (Boudaba et al., 1996) that innervate the presynaptic glutamate neurons in the PVN. Severing these projections with surgical isolation of the PVN could lead to disinhibition of the PVN glutamate interneurons.

Twenty of the norepinephrine-responsive magnocellular neu- 


\section{Biocytin}
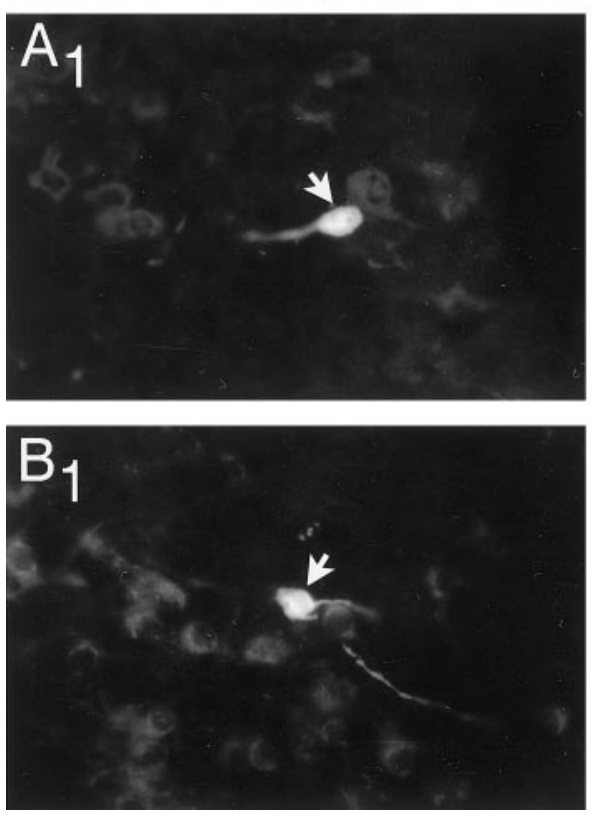

Vasopressin
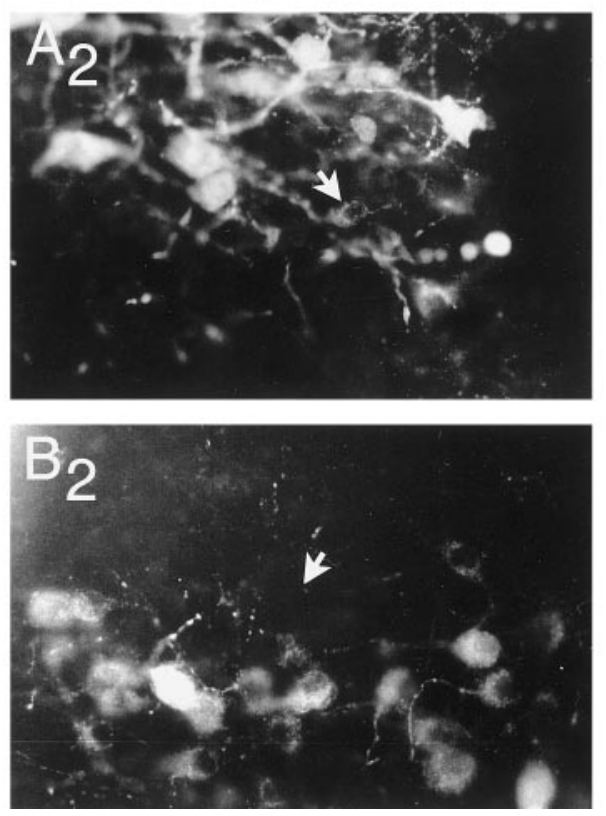

Oxytocin
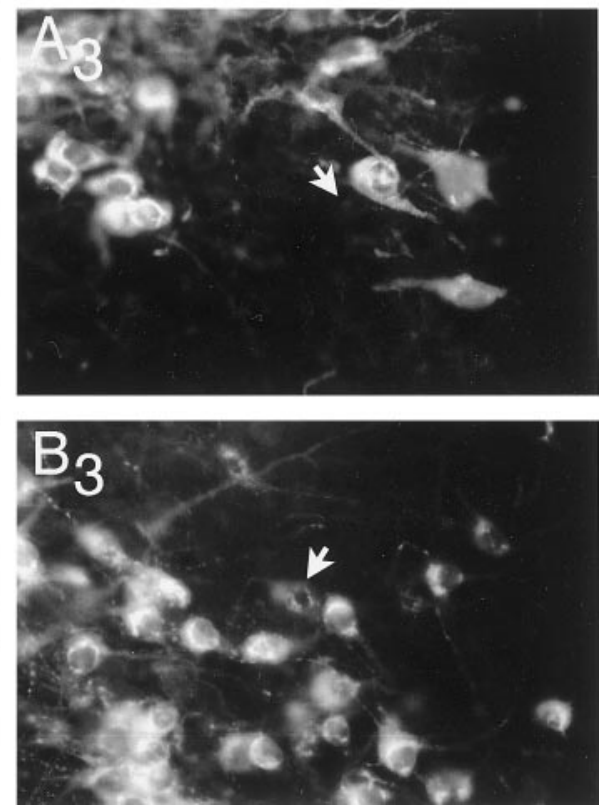

Figure 7. Immunohistochemical double-labeling of norepinephrine-responsive oxytocin and vasopressin magnocellular neurons in the PVN. Magnocellular neurons that responded to norepinephrine with an increase in EPSPs were injected with biocytin and immunohistochemically processed with antibodies to oxytocin and vasopressin-associated neurophysin. $A$, A biocytin-injected, AMCA-labeled cell $\left(A_{1}\right.$, arrow) was visualized under the AMCA filter combination (Biocytin). The same section was visualized under rhodamine filters to detect the vasopressinergic neurons (Vasopressin) and under FITC filters to see the oxytocinergic neurons (Oxytocin). This cell was immunopositive for vasopressin $\left(A_{2}\right.$, arrow $)$ and immunonegative for oxytocin $\left(A_{3}\right.$, arrow), indicating that it was a vasopressinergic magnocellular neuron. $B$, Another magnocellular neuron that responded to norepinephrine with an increase in EPSPs (recordings shown in Fig. 2$)$ and was labeled with biocytin $\left(B_{1}\right.$, arrow) was immunonegative for vasopressin $\left(B_{2}\right.$, arrow) and immunopositive for oxytocin $\left(B_{3}\right.$, arrow), indicating that it was an oxytocinergic magnocellular neuron. The FITC-oxytocin label bleeds through the UV filters used to visualize the biocytin-AMCA label but is readily distinguished from the AMCA label by its intensity and its color (seen as gray scale here). $200 \times$ magnification.

rons were identified immunohistochemically as oxytocinergic or vasopressinergic (i.e., labeled positive for one peptide and negative for the other). Approximately $63 \%$ of the neurons that responded to norepinephrine with a direct depolarization (five of eight) and $75 \%$ of the neurons that responded with an increase in EPSPs (9 of 12) were oxytocinergic, whereas the remaining cells, $37 \%$ and $25 \%$, respectively, were vasopressinergic. It is not possible from these data to ascertain whether this represents a preferential responsiveness of oxytocin neurons to norepinephrine inputs or a sampling bias in our recordings. However, it is clear that both oxytocin and vasopressin neurons of the PVN express functional $\alpha_{1}$-adrenoreceptors and receive local excitatory synaptic inputs from $\alpha_{1}$-adrenoreceptor-expressing glutamate neurons in the PVN. A schematic diagram of a proposed model circuit is presented in Figure 8.

The intranuclear origin of the glutamatergic inputs to magnocellular neurons raises the question of the identity of the presynaptic cells. There are several possibilities, including a separate population of glutamatergic interneurons, a subtype of PVN parvocellular neuron that co-expresses glutamate, and other magnocellular neurons that co-express glutamate along with vasopressin or oxytocin. In the course of this study, we recorded the responses of both putative magnocellular and putative parvocellular neurons to norepinephrine, and interestingly, very few putative parvocellular neurons $(\sim 2 \%)$ were depolarized by norepinephrine (Daftary et al., 1996) [note that all nonmagnocellular neurons located in the PVN were classified as parvocellular neurons; Hoffman et al. (1991)]. In contrast, $23 \%$ of the putative magnocellular neurons showed a relatively robust depolarization $(7.27 \pm 0.6 \mathrm{mV})$ in response to norepinephrine, as described above. This depolarization was strong enough in some of the neurons to generate a train of action potentials (Fig. $1 A$ ), which if the cells co-released glutamate would induce a robust increase in EPSPs postsynaptically, similar to the synaptic response to norepinephrine reported here. Although more experiments are necessary to determine the identity of the presynaptic neurons, our current working hypothesis is that the glutamatergic inputs to oxytocin and vasopressin neurons arise either from other magnocellular neurons or from a separate, sparsely distributed population of glutamatergic interneurons in the PVN.

Indirect noradrenergic activation of oxytocin neurons via glutamate interneurons could account for seemingly paradoxical findings from both anatomic and physiological studies. Although there is compelling evidence that noradrenergic afferents and norepinephrine play a critical role in the control of oxytocin and vasopressin release (Tribollet et al., 1978; Clarke et al., 1979; Randle et al., 1986b; Crowley and Armstrong, 1992), several immunohistochemical studies have suggested that noradrenergic inputs are less densely concentrated in intranuclear regions occupied by oxytocin neurons than those in which vasopressin neurons predominate (McNeill and Sladek, 1980; Swanson et al., 1981; Hornby and Piekut, 1987; Cunningham and Sawchenko, 1988; Ginsberg et al., 1994; although see Michaloudi et al., 1997). Our current findings provide a physiological correlate to recent observations of noradrenergic synapses directly on both oxytocin and vasopressin neuronal somata (Michaloudi et al., 1997), but 


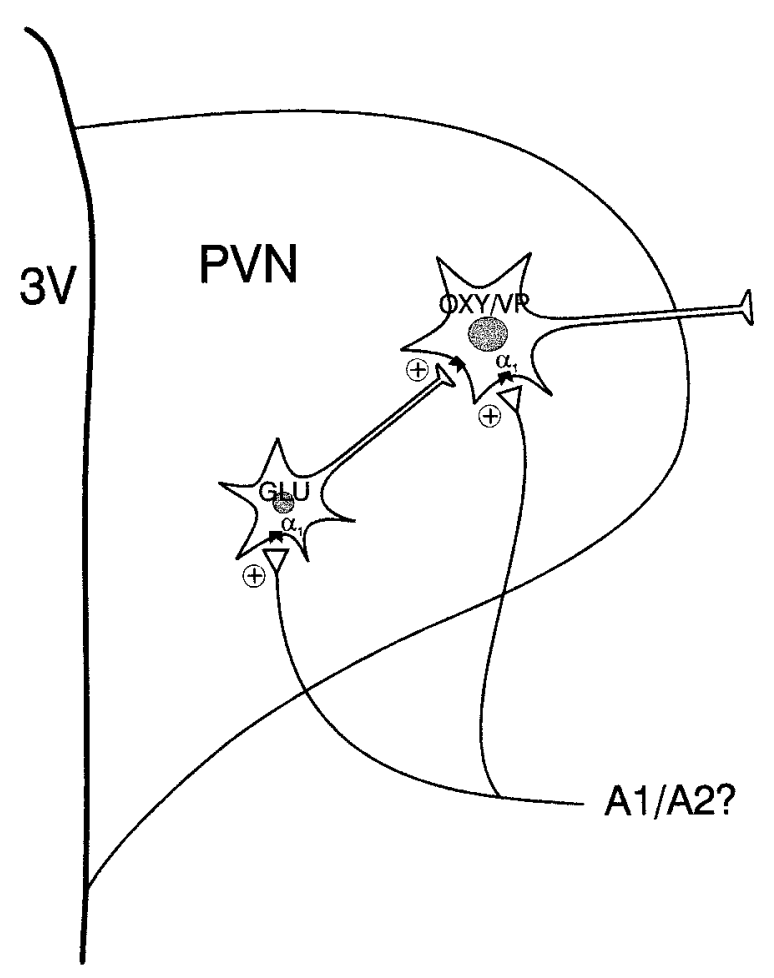

Figure 8. Model of the regulation of magnocellular neurons by norepinephrine. Norepinephrine projections, probably from the A1/A2 noradrenergic cell groups in the medulla, activate glutamatergic interneurons $(G L U)$ within the PVN that send intranuclear excitatory projections to oxytocinergic and vasopressinergic magnocellular neurons $(O X Y / V P)$. Norepinephrine acts via $\alpha_{1}$-adrenoreceptors to excite the glutamate interneurons, which leads to an increase in ionotropic receptor-mediated EPSPs in oxytocin and vasopressin neurons. PVN oxytocin and vasopressin neurons also receive direct noradrenergic inputs and express $\alpha_{1}$ adrenoreceptors. $3 \mathrm{~V}$, Third ventricle.

they also suggest that many of the oxytocin and vasopressin neurons in the PVN receive an indirect input from noradrenergic afferents by way of glutamatergic relay cells. This is consistent with the facilitatory interaction between adrenoreceptor and glutamate-receptor mechanisms on oxytocin release described in the lactating rat (Parker and Crowley, 1993b). These data also are in line with the observation that norepinephrine levels in the SON and PVN show a prolonged increase before and during the parturition-associated release of oxytocin, whereas glutamate levels rise sharply just before oxytocin release and subside rapidly (Herbison et al., 1997). Taken together, these findings suggest that noradrenergic afferents may be activated by sensory inputs in a tonic or slow phasic manner and that after a latency caused by an as-yet-unknown gating mechanism at the level of the PVN (and SON?), glutamate interneurons are stimulated to fire abruptly to trigger oxytocin neuron activation and bolus release of oxytocin. Parker and Crowley (1993b) found that the increase in oxytocin release caused by norepinephrine application in the SON in vivo was blocked by an AMPA receptor antagonist, but also that the glutamate-induced increase in oxytocin release was attenuated by an adrenoreceptor antagonist. This suggests that the situation is probably more complicated than the simple monosynaptic and disynaptic PVN circuits presented in Figure 8.

Although the role of the intranuclear glutamate circuits in the control of oxytocin and vasopressin release is not yet known, their presence provides a significant potential mechanism for the gen- eration and coordination of the patterned electrical activity seen in oxytocin and vasopressin neurons. A recent report showed that local glutamatergic circuits in hypothalamic slice cultures are capable of driving bursting activity in individual oxytocin neurons (Jourdain et al., 1998). In addition to serving as a pattern generator, a small group of glutamate interneurons could provide synchronizing inputs to oxytocin neurons in the PVN, and if these glutamatergic projections extended to the other magnocellular nuclei, they could drive the synchronous bursting activity characteristic of these neurons during the milk ejection reflex and parturition. Future studies will need to determine whether similar intranuclear glutamatergic circuits are present in lactating female rats and whether glutamatergic projections serve to interconnect the magnocellular nuclei.

\section{REFERENCES}

Altstein M, Whitnall MH, House S, Key S, Gainer H (1988) An immunochemical analysis of oxytocin and vasopressin prohormone processing in vivo. Peptides 9:87-105.

Armstrong WE, Gallagher MJ, Sladek CD (1986) Noradrenergic stimulation of supraoptic neuronal activity and vasopressin release in vitro: mediation by an alpha 1-receptor. Brain Res 365:192-197.

Ben-Barak Y, Russell JT, Whitnall MH, Ozato K, Gainer H (1985) Neurophysin in the hypothalamo-neurohypophysial system. I. Production and characterization of monoclonal antibodies. J Neurosci 5:81-97.

Benetos A, Gavras I, Gavras H (1986) Norepinephrine applied in the paraventricular hypothalamic nucleus stimulates vasopressin release. Brain Res 381:322-326.

Boudaba C, Szabo K, Tasker JG (1996) Physiological mapping of local inhibitory inputs to the hypothalamic paraventricular nucleus. J Neurosci 16:7151-7160.

Boudaba C, Schrader LA, Tasker JG (1997) Physiological evidence for local excitatory synaptic circuits in the rat hypothalamus. J Neurophysiol 77:3396-3400.

Bourque CW, Renaud LP (1991) Membrane properties of rat magnocellular neuroendocrine cells in vivo. Brain Res 540:349-352.

Bridges TE, Hillhouse EW, Jones MT (1976) The effect of dopamine on neurohypophysial hormone release in vivo and from the rat neural lobe and hypothalamus in vitro. J Physiol (Lond) 260:647-666.

Christian EP, Dudek FE (1988) Characteristics of local excitatory circuits studied with glutamate microapplication in the CA3 area of rat hippocampal slices. J Neurophysiol 59:90-109.

Clarke G, Lincoln DW, Merrick LP (1979) Dopaminergic control of oxytocin release in lactating rats. J Endocrinol 83:409-420.

Crowley WR, Armstrong WE (1992) Neurochemical regulation of oxytocin secretion in lactation. Endocr Rev 13:33-65.

Crowley WR, Shyr SW, Kacsoh B, Grosvenor CE (1987) Evidence for stimulatory noradrenergic and inhibitory dopaminergic regulation of oxytocin release in the lactating rat. Endocrinology 121:14-20.

Cunningham Jr ET, Sawchenko PE (1988) Anatomical specificity of noradrenergic inputs to the paraventricular and supraoptic nuclei of the rat hypothalamus. J Comp Neurol 274:60-76.

Daftary SS, Szabó K, Tasker JG (1996) The effects of norepinephrine on the electrical properties of identified neurons of the hypothalamic paraventricular nucleus. Soc Neurosci Abstr 22:2014.

Day TA, Renaud LP (1984) Electrophysiological evidence that noradrenergic afferents selectively facilitate the activity of supraoptic vasopressin neurons. Brain Res 303:233-240.

Day TA, Sibbald JR (1988) Direct catecholaminergic projection from nucleus tractus solitarii to supraoptic nucleus. Brain Res 454:387-392.

Day TA, Ferguson AV, Renaud LP (1984) Facilitatory influence of noradrenergic afferents on the excitability of rat paraventricular nucleus neurosecretory cells. J Physiol (Lond) 355:237-249.

Ginsberg SD, Hof PR, Young WG, Morrison JH (1994) Noradrenergic innervation of vasopressin- and oxytocin-containing neurons in the hypothalamic paraventricular nucleus of the macaque monkey: quantitative analysis using double-label immunohistochemistry and confocal laser microscopy. J Comp Neurol 341:476-491.

Herbison AE, Voisin DL, Douglas AJ, Chapman C (1997) Profile of monoamine and excitatory amino acid release in rat supraoptic nucleus over parturition. Endocrinology 138:33-40.

Hoffman NW, Tasker JG, Dudek FE (1991) Immunohistochemical dif- 
ferentiation of electrophysiologically defined neuronal populations in the region of the rat hypothalamic paraventricular nucleus. J Comp Neurol 307:405-416.

Hornby PJ, Piekut DT (1987) Catecholamine distribution and relationship to magnocellular neurons in the paraventricular nucleus of the rat. Cell Tissue Res 248:239-246.

Jourdain P, Israel J-M, Dupouy B, Oliet SH, Allard M, Vitiello S, Theodosis DT, Poulain DA (1998) Evidence for a hypothalamic oxytocin-sensitive pattern-generating network governing oxytocin neurons in vitro. J Neurosci 18:6641-6649.

Kim YI, Dudley CA, Moss RL (1989) Re-evaluation of the effects of norepinephrine on the single-unit activity of paraventricular neurosecretory neurons. Neurosci Lett 97:103-110.

Legendre P, Poulain DA (1992) Intrinsic mechanisms involved in the electrophysiological properties of the vasopressin-releasing neurons of the hypothalamus. Prog Neurobiol 38:1-17.

Lena C, Changeux JP, Mulle C (1993) Evidence for "preterminal" nicotinic receptors on GABAergic axons in the rat interpeduncular nucleus. J Neurosci 13:2680-2688.

McNeill TH, Sladek Jr JR (1980) Simultaneous monoamine histofluorescence and neuropeptide immunocytochemistry. II. Correlative distribution of catecholamine varicosities and magnocellular neurosecretory neurons in the rat supraoptic and paraventricular nuclei. J Comp Neurol 193:1023-1033.

Michaloudi HC, el Majdoubi M, Poulain DA, Papadopoulos GC, Theodosis DT (1997) The noradrenergic innervation of identified hypothalamic magnocellular somata and its contribution to lactation-induced synaptic plasticity. J Neuroendocrinol 9:17-23.

Parker SL, Crowley WR (1993a) Stimulation of oxytocin release in the lactating rat by central excitatory amino acid mechanisms: evidence for specific involvement of $\mathrm{R}, \mathrm{S}-\alpha$-amino-3-hydroxy-5-methylisoxazole-4propionic acid-sensitive glutamate receptors. Endocrinology 133: $2847-2854$.
Parker SL, Crowley WR (1993b) Stimulation of oxytocin release in the lactating rat by a central interaction of $\alpha 1$-adrenergic and $\alpha$-amino-3hydroxy-5-methylisoxazole-4-propionic acid-sensitive excitatory amino acid mechanisms. Endocrinology 133:2855-2860.

Randle JC, Bourque CW, Renaud LP (1986a) $\alpha 1$-adrenergic receptor activation depolarizes rat supraoptic neurosecretory neurons in vitro. Am J Physiol 251:R569-R574.

Randle JC, Mazurek M, Kneifel D, Dufresne J, Renaud LP (1986b) $\alpha 1$-adrenergic receptor activation releases vasopressin and oxytocin from perfused rat hypothalamic explants. Neurosci Lett 65:219-223.

Schrader LA, Tasker JG (1997) Presynaptic modulation by metabotropic glutamate receptors of excitatory and inhibitory synaptic inputs to hypothalamic magnocellular neurons. J Neurophysiol 77:527-536.

Swanson LW, Sawchenko PE, Berod A, Hartman BK, Helle KB, Vanorden DE (1981) An immunohistochemical study of the organization of catecholaminergic cells and terminal fields in the paraventricular and supraoptic nuclei of the hypothalamus. J Comp Neurol 196:271-285.

Tanaka J, Kaba H, Saito H, Seto K (1985) Inputs from the A1 noradrenergic region to hypothalamic paraventricular neurons in the rat. Brain Res 335:368-371.

Tasker JG, Dudek FE (1991) Electrophysiological properties of neurones in the region of the paraventricular nucleus in slices of rat hypothalamus. J Physiol (Lond) 434:271-293.

Tribollet E, Clarke G, Dreifuss JJ, Lincoln DW (1978) The role of central adrenergic receptors in the reflex release of oxytocin. Brain Res 142:69-84.

Van Huysse JW, Bealer SL (1991) Central nervous system norepinephrine release during hypotension and hyperosmolality in conscious rats. Am J Physiol 260:R1071-R1076.

Willoughby JO, Jervois PM, Menadue MF, Blessing WW (1987) Noradrenaline, by activation of $\alpha$-1-adrenoceptors in the region of the supraoptic nucleus, causes secretion of vasopressin in the unanaesthetized rat. Neuroendocrinology 45:219-226. 